\title{
Critical Data Identification, Information Communication, and Readily Available Knowledge Base for Effectively Mitigating Impact of Potentially- Hazardous-Asteroid/Object (PHA/PHO)
}

\author{
Chaowei Phil Yang*, GMU/NASA Goddard \& NSF Spatiotemporal Innovation Center \\ Luisa Fernanda Zambrano-Marin, Arecibo Observatory (UCF/FSI) \\ Kyla Carte, NSF Spatiotemporal Innovation Center/GMU \\ Amanda K. Davis, Johns Hopkins Applied Physics Laboratory \\ Dawn M. Graninger, Johns Hopkins Applied Physics Laboratory \\ Kevin Greenaugh, National Nuclear Security Administration \\ Catherine McCarthy, Georgetown University \\ Catherine Suzanne Plesko, Los Alamos National Lab \\ Bruno Victorino Sarli, Alcyon Technical Services (JV), LLC \\ Dexuan Sha, NSF Spatiotemporal Innovation Center/GMU \\ Ishan Shams, NSF Spatiotemporal Innovation Center/GMU \\ Dipak Srinivasan, Johns Hopkins Applied Physics Laboratory \\ Angela Stickle, Johns Hopkins Applied Physics Laboratory \\ Megan Bruck Syal, Lawrence Livermore, National Lab \\ Allen Zhong, NSF Spatiotemporal Innovation Center/GMU \\ *Leading author contact information: cyang3@gmu.edu, 571-235-1827
}

Acknowledgements: Time didn't allow an official NASA approval, colleagues contributed also include

Ronald Leung, NASA Goddard Space Flight Center

Ruthan Lewis, NASA Goddard Space Flight Center

Joshua R. Lyzhoft, NASA Goddard Space Flight Center

Joseph Nuth, NASA Goddard Space Flight Center

LIoyd R. Purves, NASA Goddard Space Flight Center

Lorien Wheeler, NASA Ames Research Center

Jessie L. Dotson, NASA Ames Research Center

\begin{abstract}
A Potentially Hazardous Asteroid/Object (PHA/PHO) colliding with Earth is a rare but possibly catastrophic event. Recent research has devised mitigation strategies for PHA/PHO's. Like the once-in-100-years COVID-19 pandemic, a PHA/PHO collision, particularly one with a short warning time, will likely challenge human society along several fronts including the identification of critical data sources, communication among different stakeholders and computer systems, and utilization of readily available knowledge to devise efficient mitigation strategies. Without the right data, effective communication, and well-
\end{abstract}


managed knowledge to support mitigation strategies and actions, the results will be confusion, missed opportunities, and inexorable anxieties.

This white paper reviews the current approaches for organizational engagement, data identification, communication, and recommends commencing a formulated effort within the planetary defense (PD) community to better identify gaps in our capability to efficiently mitigate the impacts of PHA/PHO. Specifically, the Planetary Defense (PD) community should address the efficient identification of and open access to critical data, interoperability of relevant systems (machine-to-machine communication), means for effective communication among stakeholders, and rapid utilization of available knowledge to support decision making.

\section{Problem Description}

Sixty-six million years ago a large impactor between 10 to 90 kilometers in diameter struck Chicxulub, Mexico [5]. The impact shockwave ejected large amounts of rock and soil into the Earth's atmosphere, causing global climate change [5], which likely was responsible for the mass extinction event of large animals including the dinosaurs [6, 10]. Although a 10-km size impact is infrequent, the considerable damage from more frequent impacts like the Chelyabinsk collision on Feb 15, 2013 by a 20-meter asteroid show the need to address the risk that potentially hazardous asteroids (PHA) collisions pose for the Earth [20].

In response to such a threat, the United States Government passed the George E. Brown Jr., Near-Earth Object Survey Act tasking NASA to detect, track, catalog and characterize $90 \%$ of all Near Earth Asteroids larger than 140 meters [17]. Following this initiative, large volumes of data, information, and knowledge are being developed to produce mitigation strategies for a PHA threat [22]. However, the three key pillars for any successful mitigation plan (i.e., data, information, knowledge) are currently distributed across different branches of global organizations [23]. A consequence of this fragmentation is that research by many different Planetary Defense (PD) experts is neither integrated nor synchronous.

Effective integration of the data, information, and knowledge in a seamless fashion, as well as fast communication among experts, agencies, and organizations, are priorities for mitigating a potential threat to planet Earth by PHA. There are three relevant aspects for integrating the resources and producing cohesive solutions to PD mitigation.

1) Data/Information Identification: Identifying and processing critical data regarding the potential threat an asteroid present, such as characterization of the PHA's spatiotemporal occurrence, is the first step to any PD effort. Recognition of the capabilities within different organizations for data processing and simulation is the pinnacle of any mitigation plan. The objective is to identify the information interconnections to support coordinated observations, verify the observations (or extensions of physics-based and other models), and produce appropriate mitigation plans.

2) Communication and International Engagement: One key component of threat mitigation is ensuring that national efforts leverage international capabilities for observation, data processing, simulation development, knowledge sharing, and actionable solutions. Some PD (related) missions have incorporated international collaboration, notably OSIRIS-Rex [12] and DART [15]. It is a priority to establish a communication protocol among countries with mitigation capabilities to avoid the uncertainties resulting from different countries executing their own mitigations to the same threat. 
3) Knowledge Base: The calculated probability of a PHA impacting Earth within the next five to ten years has decreased over time because of ongoing efforts to catalogue and better understand large space rocks. However, prediction for the next 100 to 300 years has a higher level of uncertainty. This paper proposes the creation of a data/knowledge base which integrates research results and the expert knowledge, two critical components, for mitigating future PHA threats with efficiency and a higher probably of success [1, 23].

\section{Objectives}

The objective of this white paper is to identify the gaps in data/information identification, communication, international engagement, and a sustainable knowledge base. More specifically, this paper endeavors to identify the existing resources and analyze connectivity/ communication channels, and then use the resulting findings to propose a more integrative and responsive PD surveillance, identification, analysis, and mitigation architecture (Table 1) to significantly improve our collaborative response to a PHA.

Table 1: Needs, Goals, and Gaps

\begin{tabular}{|c|c|c|c|}
\hline Needs & Goals & Gaps & Comments \\
\hline $\begin{array}{c}\text { Identification of key US } \\
\text { national/international } \\
\text { level participants. } \\
\text { Specifically, relevant } \\
\text { information producers, } \\
\text { consumers, and products }\end{array}$ & $\begin{array}{l}\text { Produce functional flow } \\
\text { network “diagram” depicting } \\
\text { information, response time of } \\
\text { products, and potential } \\
\text { communication pathways }\end{array}$ & $\begin{array}{l}\text { Identify gaps for } \\
\text { coordinating outputs at } \\
\text { the national level and } \\
\text { expand to the } \\
\text { international level }\end{array}$ & $\begin{array}{c}\text { This is not an } \\
\text { "organizational chart" } \\
\text { but a functions and } \\
\text { flow connectivity } \\
\text { diagram }\end{array}$ \\
\hline $\begin{array}{l}\text { Data/information } \\
\text { identification and } \\
\text { integration }\end{array}$ & $\begin{array}{l}\text { Identify the workflow of data } \\
\text { and information extraction } \\
\text { methods and tools }\end{array}$ & $\begin{array}{c}\text { Identify the solution gap } \\
\text { for data/information and } \\
\text { integration }\end{array}$ & $\begin{array}{c}\text { Consider } \\
\text { heterogeneity, } \\
\text { metadata, ontology, } \\
\text { and knowledge for } \\
\text { smart discovery and } \\
\text { integration }\end{array}$ \\
\hline $\begin{array}{l}\text { Communication } \\
\text { analytics }\end{array}$ & $\begin{array}{l}\text { Identify the existing } \\
\text { visualization tools for different } \\
\text { stakeholders }\end{array}$ & $\begin{array}{l}\text { Identify the gap for } \\
\text { effective communication } \\
\text { of information and } \\
\text { knowledge among the } \\
\text { stakeholders and } \\
\text { decision makers }\end{array}$ & $\begin{array}{l}\text { Consider the } \\
\text { interactive } \\
\text { visualization of } \\
\text { different aspects } \\
\text { comprehensively for } \\
\text { easy understanding. }\end{array}$ \\
\hline $\begin{array}{l}\text { Identify available } \\
\text { knowledge and gaps }\end{array}$ & $\begin{array}{l}\text { Maintain up-to-date functions } \\
\text { and proper maintenance of a } \\
\text { knowledge base }\end{array}$ & $\begin{array}{l}\text { Perform checks of the } \\
\text { functions to identify gaps } \\
\text { of the knowledge base } \\
\text { and its data for validity }\end{array}$ & $\begin{array}{l}\text { Improve functions and } \\
\text { maintain a knowledge } \\
\text { base }\end{array}$ \\
\hline $\begin{array}{l}\text { Timely response to high } \\
\text { consequential threat }\end{array}$ & $\begin{array}{l}\text { Generate options / scenarios } \\
\text { within timeframe of weeks or } \\
\text { days based on established } \\
\text { knowledge base }\end{array}$ & $\begin{array}{l}\text { Identify response "bottle } \\
\text { necks”, gaps in } \\
\text { information needed to } \\
\text { produce scenarios } \\
\text { (decisions), response } \\
\text { time “delays / lags", and } \\
\text { key analytical tools } \\
\text { needed (e.g., models, } \\
\text { tests, data) }\end{array}$ & $\begin{array}{c}\text { Learn from recent } \\
\text { encounter } \\
\text { characteristics of } \\
\text { pandemic COVID-19 }\end{array}$ \\
\hline
\end{tabular}




\section{Literature Review and Current Status}

The proposed actions directly correlate to the National Near-Earth Object (NEO) Preparedness Strategy and Action Plan [8], i.e., Goal 2, modeling, prediction, and information integration; Goal 4, international collaboration; and Goal 5, NEO impact emergency procedures and action protocols. Three points of focus are critical to achieving the three goals: 1) data identification, 2) information communication, and 3) a knowledge base. Each is discussed herein with respect to a conceptual architecture and gap analyses.

\subsection{Workflow and Overall Conceptual Architecture}

Many US national organizations are engaged in PD efforts (Figure 1a), besides which other national and international organizations play critical roles in different segments of PD mitigation effort. Without a structured framework for information access and flow, an efficient, streamlined, and consolidated effort amongst the various organizations is at risk. Scattered and unorganized information is a significant liability at the time of crisis, resulting in inefficient processes and decisions on incomplete data. The National Aeronautics and Space Administration (NASA) Goddard and the National Science Foundation (NSF) Spatiotemporal Innovation Center (chartered by NSF in 2013 and renewed in 2017, and sponsored by NASA, USGS, State Dept., Defense Dept. and others) proposed a non-exclusive conceptual framework (Figure 1b) to integrate dispersed and diverse information residing at different organizations within the United States[1]. Such a conceptual architecture should be detailed as a reference architecture and include international organizations to facilitate the data, information and knowledge process in PD mitigation.
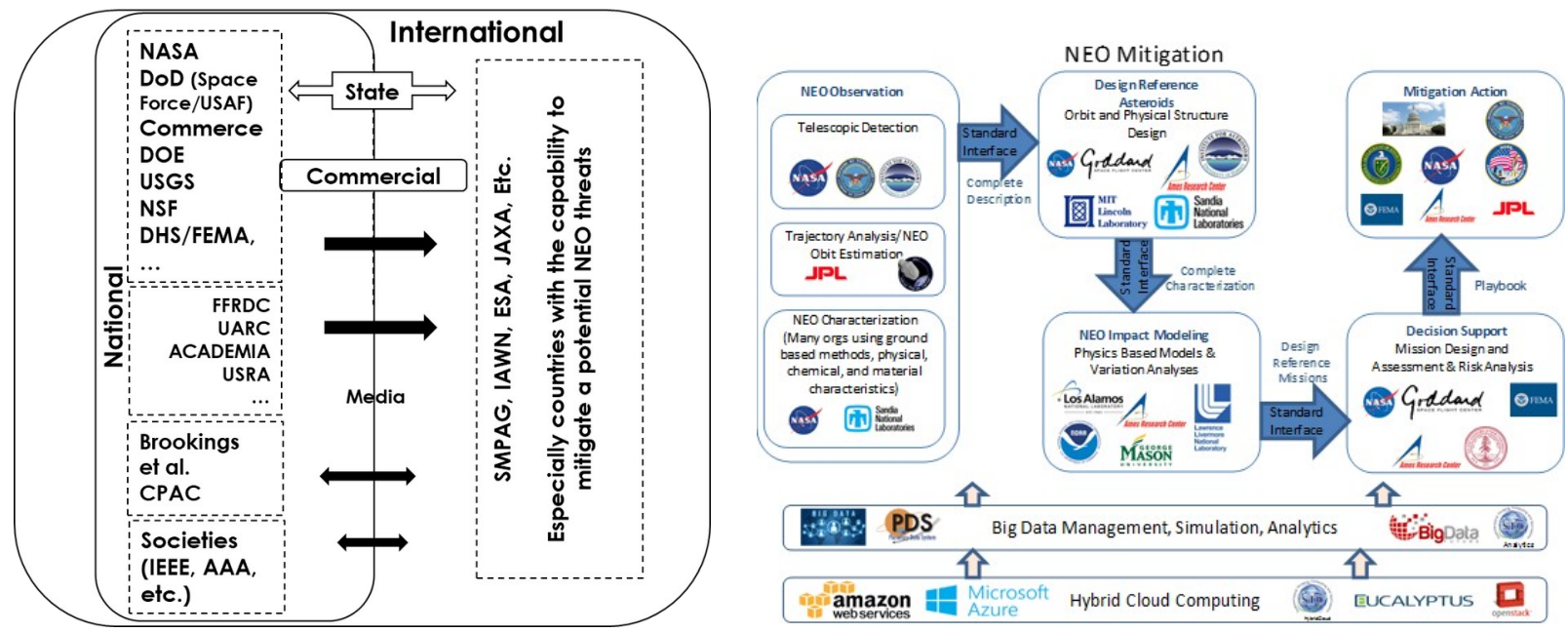

Figure 1a (left): Organizations and their potential interactions for mitigating NEO threat; $1 \mathrm{~b}$ (right): A non-exclusive architecture for the NEO mitigation process [1].

\subsection{Data and Information Sources}

Data identification: Data (from observations or analytics) are fed in analytical tools for obtaining the necessary information to support decision making. For example, researchers used prior meteorite impact data plus a NEO population orbit model to compute probable escape routes of the main asteroid belt and the cometary region [7]. The Arcetri Near Earth Object Precovery Program (ANEOPP) identifies NEOs from previous "archival materials" [3]. In addition, ground based planetary radar systems such as those at Arecibo Observatory and 
Goldstone Observatory [28] contribute high precision physical and orbital characterization of PHA for NASA projects and missions, including OSIRIS Rex, LRO, Cassini, Clementine, Mars Exploration Rovers (MERs), InSight, and MESSENGER [14, 25]. Additionally, a comprehensive catalogue of mission instrumentation, critical to characterize a PHA for producing solutions to mitigate impact, should also be included in a PD data source for future access. NASA GSFC and NSF's Spatiotemporal Innovation Center currently host pertinent PD instrument and mission tables. Incorporating these and similar tables into a database could improve mitigation capabilities and assist in identifying instrument advancements needed for reaching even higher expectations mitigation capabilities.

Data Fusion Strategies: The 2020 global COVID-19 health crisis motivated the development of data fusion strategies to benefit future scientists with readily available knowledge using the same reference framework [30]. Although it is possible to eventually produce outputs consistent with all relevant and available data, during a crisis it can become challenging to integrate information from many heterogenous sources, especially when the relevant data model is very complex. Intriguing research questions in the planetary domain can be actively answered with higher accuracy by incorporating data fusion techniques. An integrated data-infused resource encourages researchers to share findings within the PD community. Such an integrated strategy provides benefits including a) a secure and more reliable environment; b) an effective way of sharing information and resources; and c) a capability to access data at any time.

\subsection{Information Communication}

The National Near-Earth Object Preparedness Strategy and Action Plan [8] emphasizes the importance of international communication and collaboration among policy and decision makers [2]. Information and communication technologies could help enhance public awareness of potential PHA impacts [25], especially for understanding risks [21]. Visualization, including maps, charts, and diagrams, is one key for communicating data among different PD stakeholders to present complex information in an easily understandable approach. Visualizations help us understand why things are happening, as well as compare different patterns and trends that could inform future outcomes, which could help us better recognize the PHA threat [19]. In the U.S. there are several ground-based NEO-search programs to monitor, track, and discover NEOs with the aid of visual analytical tools [29]. In addition, many organizations have created their own PD visual tool to track and monitor the solar system, NEOs, and the health of other planets (Table 2).

Table 2: Analytical visualization examples that can be adjusted for PD

\begin{tabular}{|c|c|}
\hline Tools & Description \\
\hline $\begin{array}{l}\text { NASA Eyes on the } \\
\text { SOLAR SYSTEM }\end{array}$ & $\begin{array}{l}\text { Visualization lets user travel throughout the solar system and fly alongside the spacecraft } \\
\text { - both current and historic [18]. }\end{array}$ \\
\hline Universe Sandbox & $\begin{array}{l}\text { Physics-based space simulator merges gravity, climate, collision, and material } \\
\text { interactions to reveal the beauty of our universe and the fragility of our planet [24]. }\end{array}$ \\
\hline $\begin{array}{l}\text { Kerbal } \\
\text { Space Program }\end{array}$ & $\begin{array}{l}\text { Space program simulator allows us to build and fly rockets and space planes, get them } \\
\text { into orbit, and perform scientific experiments from space. During its development, } \\
\text { NASA collaborated with KSP's developers to create an in-game mission mirroring } \\
\text { NASA's Asteroid Redirect Mission [9]. }\end{array}$ \\
\hline
\end{tabular}

International collaboration is key to a successful PD mitigation [11, 27] as evidenced by the Roadmap for EArth Defense Initiative (READI) Project, a collaboration among 17 countries, for defending against short-warning impacts of PHA [16]. Two leading groups that assist in 
improving joint PD efforts among nations are the International Asteroid Warning Network (IAWN) and the Space Mission Planning Advisory Group (SMPAG) [11].

Unlike these purely human-to-human communications, machine-to-machine communications are not well investigated. Most systems are developed in a closed (stove-pipe) environment without considering standards for data and protocols for communication across systems. Opening the systems and standardizing the data formats, communication protocols are urgently needed.

\subsection{Knowledge Base}

The National Near-Earth Object Preparedness Strategy and Action Plan [8] urges the United States to lead an international effort to track and characterize PHA collision threats, protect against damaging impacts, and respond to and recover from them. Such a comprehensive PHA impact response requires the integration of human knowledge from different national and international organizations using a knowledge base [23]. Various data types and formats, wellorganized entities, concrete field and high-level knowledge [1] can be managed and reasoned in a comprehensive mapped ontology which includes objective concept, property, and instance in the PD knowledge domain [13]. The architecture of the PD knowledge base (Figure 2a) could contain several parts including data acquisition, ontology processing, and knowledge extraction. A knowledge base framework structured by domain experts and filled with multi-source data could produce entity, relationship, and other information insights, conduct scenario simulations, and generate customized solutions for PD mitigation. For instance, a visualization shows the mitigation needs of missions, relevant instruments, participation organizations, and simulation models (Figure 2b). With such implementation, many scenarios and solutions can be simulated based on a knowledge framework. Moreover, knowledge management, PD corpus, and customized reports can be improved by a PD knowledge base [23].
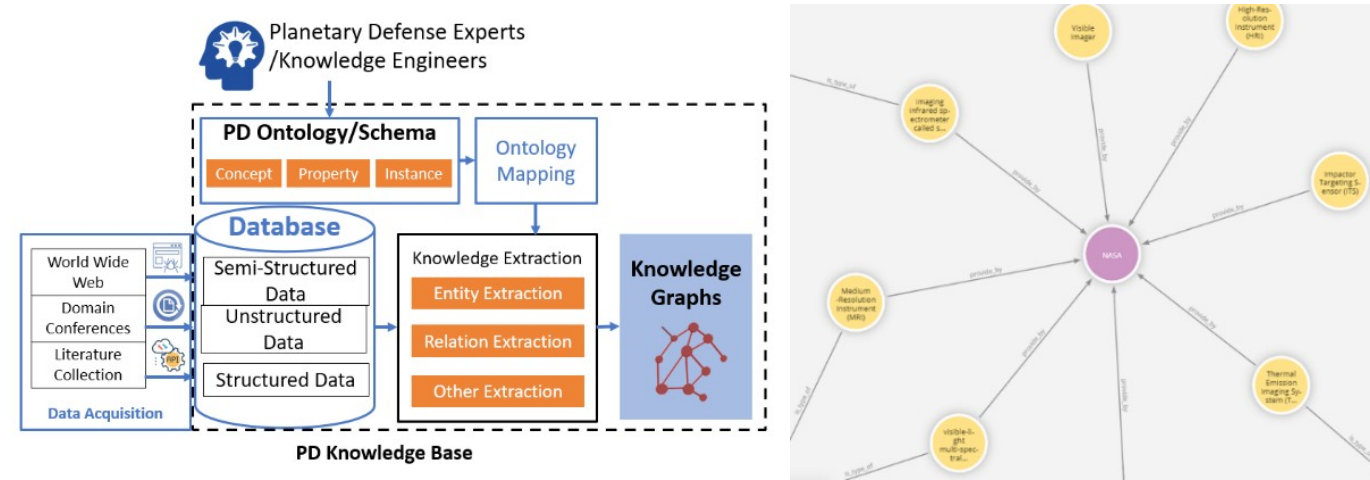

Figure 2a (left). A potential knowledge architecture; $2 b$ (right) A knowledge navigation and reasoning graph for PD (with information identification as an example)

\subsection{Gap Identification Methodology}

The NASA GSFC and NSF Spatiotemporal Innovation Center analyzed the current status and needs to support the functions and processes for the mitigation of a potential PHA impact in a sustainable and extensible system of systems conceptual infrastructure (Figure 1b)[1]. After an extensive study of the organizational assets that could contribute to PHA mitigation around the world and especially those within the United States (e.g., NASA, National Nuclear Security Administration/NNSA), the conceptual infrastructure identifies a process for mitigating PHA impacts using observation and characterization of reference asteroids, impact modeling, mission design, in order to support decisions for mitigation actions [1]. This architecture could help 
clarify the step-by-step process of the complex PHA mitigation process to assist scientists and government officials in understanding/improving the information flow. Based on input by the PD community, several gaps and recommendations are summarized in the following section.

\section{Gap Summary and Recommendations for the Next Decadal Development}

\section{Data identification:}

- Compile a (virtual) database (DB) containing critical PD components based on existing assets (e.g., Jet Propulsion Laboratory/JPL Horizons and PD Mitigation portal [23]). This database collects object characterization data, impact predictions, and national and international emergency response plans, as well as allowing for effective communication and proper data management.

- $\quad$ NEO DBs: Existing DBs (e.g., International Astronomical Union Minor Planet Center database and the JPL's Small Body Database) have pioneered efforts in data collection and sharing. A more robust system is needed where multiple wavelength observations are logged, and data seamlessly linked with standardized data formats and communication protocols.

- $\quad$ PD instrument and mission DBs: Different types of PD missions (i.e. kinetic impactors, solar sails, tether bodies, seek orbit diversion) have different instruments, different payloads, and different needs for launch systems. A comprehensive database for instruments and missions is needed as a reference to design new PD missions within a limited amount of time using the current technologies at time of execution.

- Publication Database: Peer reviewed publications are critical source for PD knowledge. A database that captures the publication is proposed to preserve and make readily available the research, validations, missions, and other components for a potential PD mitigation.

Information communication: The development of a communication reference architecture is proposed focusing on communication standards and the interfaces for machines, models, information systems, and decision makers. International collaboration is key for leveraging the latest PHA mitigation advancements from different countries. Increased cooperation among countries for PD is critical for an effective strategy to survive larger asteroid impacts.

Knowledge base: A knowledge base warrants construction to increase the efficiency and quality of PHA mitigation strategies. With the knowledge base, automatic or semi-automatic knowledge extraction techniques and smart discovery engines can be developed, resulting in semiautomatous simulations and modeling of different PHA mitigation inputs. Such a knowledge base will help minimize and identify key PHA mitigation case studies focusing on potential mitigation solutions.

\section{$5 \quad$ References Cited}

[1] Bambacus, M., et al., 2017. A Knowledge Framework for Smart Discovery of Planetary Defense Resources. Planetary Defense Conference. 2017.

[2] Billings, L., 2015. Words matter: A call for responsible communication about asteroid impact hazards and plans for planetary defense. Space Policy, 33, pp.8-12.

[3]Boattini, A, et al., (2001). The Arcetri NEO Precovery Program. ASTRONOMY \& ASTROPHYSICS. 375(1), p293-307. Available from: 10.1051/0004-6361:20010825

[5] Collins, G.S., et al., 2020. A steeply-inclined trajectory for the Chicxulub impact. Nature communications, 11(1), pp.1-10.

[6] Durand-Manterola, H.J. and Cordero-Tercero, G., 2014. Assessments of the energy, mass and size of the Chicxulub Impactor. arXiv preprint arXiv:1403.6391. 
[7] Granvik, M and Brown, P, (2018). Identification of meteorite source regions in the Solar System. ICARUS. 311, p271-287. Available from: 10.1016/j.icarus.2018412

[8] Whitehouse, (2018). National Near-Earth Object Preparedness Strategy And Action Plan. Retrieved from https://www.whitehouse.gov/wp-content/uploads/2018/06/National-Near-EarthObject-Preparedness-Strategy-and-Action-Plan-23-pages-1MB.pdf

[9] Kerbal Space Program - Create and Manage Your Own Space Program Available online: https://www.kerbalspaceprogram.com/ (accessed on Feb 7, 2019).

[10] Kerr, R.A., 1996. A piece of the dinosaur killer found? Science, 271(5257), 1806-1806.

[11] Kofler, R., et al., 2019. International coordination on planetary defence: The work of the IAWN and the SMPAG. Acta Astronautica, 156, 409-415.

[12] Lauretta, D.S., et al., 2017. OSIRIS-REx: sample return from asteroid (101955) Bennu. Space Science Reviews, 212, 925-984.

[13] Lehmann, J., et al., (2015). DBpedia - A large-scale, multilingual knowledge base extracted from Wikipedia. Semantic Web, [online] 6(2), 167-195.

[14] Meisner, AM, Lang, D, Schlegel, DJ, (2017). FULL-DEPTH COADDS OF THE WISE AND FIRST-YEAR NEOWISE-REACTIVATION IMAGES. ASTRONOMICAL JOURNAL. 153, Available from: 10.3847/1538-3881/153/1/38

[15] Michel, P., et al., 2018. European component of the AIDA mission to a binary asteroid: Characterization and interpretation of the impact of the DART mission. Advances in Space Research, 62, pp.2261-2272.

[16] Nambiar, S.P., et al., 2016, March. Architecture for mitigating short-term warning cosmic threats: READI project. In 2016 IEEE Aerospace Conference (pp. 1-11). IEEE.

[17] NASA, 2005. National Aeronautics and Space Administration Authorization Act of 2005, https://www.govinfo.gov/content/pkg/PLAW-109publ155/pdf/PLAW-109publ155.pdf last accessed on Aug.31, 2020.

[18] NASA’s Eyes Available online: https://eyes.nasa.gov/ (accessed on Feb 7, 2019).

[19] Planetary Defense | The Planetary Society Available online: http://www.planetary.org/explore/projects/planetary-defense/ (accessed on Feb 7, 2019).

[20] Popova, O.P., et al., 2013. Chelyabinsk airburst, damage assessment, meteorite recovery, and characterization. Science, 342(6162), pp.1069-1073.

[21] Race, M. (2015). Understanding Risk Perceptions and Public Information Needs from the Bottom-Up: Important Elements for Managing Responses to Hazardous NEO's. Frascati: Planetary Defense Conference. 2015.

[22] Schmidt, N. ed., 2018. Planetary Defense: Global Collaboration for Defending Earth from Asteroids and Comets. Springer.

[23] Shams, I., et al., 2019. Planetary Defense Mitigation Gateway: A One-Stop Gateway for Pertinent PD-Related Contents. Data, 4, p.47.

[24] Universe Sandbox Available online: http://universesandbox.com/ (accessed on Feb 7, 2019). [25]Usikov, D.A., 2013. Information and Communication Technologies (ICT) as keys to the enhancement of public awareness about potential earth impacts. Acta Astronautica, 90: 173-179. [27] Pelton, J.N., 2019. Space weapons, the threat of war in space and planetary defense. In Space 2.0 (pp. 115-128). Springer, Cham.

[28] Cho, et al., 1996. High-resolution stratospheric dynamics measurements with the NASA/JPL Goldstone Solar System Radar. Geophysical research letters, 23, 1909-1912. 
[29]Paek, SW, et. al., 2020. Optimization and decision-making framework for multi-staged asteroid deflection campaigns under epistemic uncertainties.ACTA ASTRONAUTICA,167:2341.

[30] Hu, T., et al., 2020. Building an Open Resources Repository for COVID-19 Research, Data and Information Management, 4(3), 130-147. 